\title{
Estimating survival probability using the terrestrial extinction history for the search for extraterrestrial life
}

\author{
Kohji Tsumura
}

Several exoplanets have been discovered to date, and the next step is the search for extraterrestrial life. However, it is difficult to estimate the number of life-bearing exoplanets because our only template is based on life on Earth. In this paper, a new approach is introduced to estimate the probability that life on Earth has survived from birth to the present based on its terrestrial extinction history. A histogram of the extinction intensity during the Phanerozoic Eon is modeled effectively with a log-normal function, supporting the idea that terrestrial extinction is a random multiplicative process. Assuming that the fitted function is a probability density function of extinction intensity per unit time, the estimated survival probability of life on Earth is $\sim 0.15$ from the beginning of life to the present. This value can be a constraint on $f_{i}$ in the Drake equation, which contributes to estimating the number of life-bearing exoplanets.

Since the first discovery of the exoplanets in the $1990 \mathrm{~s}^{1,2}$, expectations for the discovery of extraterrestrial life have increased. The most likely outcomes are the discovery of traces of life in our Solar System such as Mars, Europa or Enceladus via in-situ explorations made by spacecrafts, or the discovery of biosignatures on exoplanets in their host star's habitable zones via astronomical high-resolution spectroscopic observations. On the other hand, there is a slight possibility that an advanced civilization will be found before these discoveries are made. For example, the Square Kilometer Array (SKA), which is the international radio telescope project currently under development, will be capable of detecting leakage emissions from Earth-level civilizations within $100 \mathrm{pc}^{3}$. Recently, the Five-hundred-meter Aperture Spherical radio Telescope (FAST) in China has been conducting Search for Extraterrestrial Intelligence (SETI) observations ${ }^{4}$. Therefore, estimating the number of planets with extraterrestrial life in our Milky Way galaxy is important.

One way to make this estimate is to use the Drake equation, which is the famous algebraic expression for quantifying the number of communicative civilizations in our Galaxy ${ }^{5}$. The Drake equation is generally expressed $\mathrm{as}^{6}$ :

$$
N=R_{*} \cdot f_{p} \cdot n_{e} \cdot f_{l} \cdot f_{i} \cdot f_{c} \cdot L
$$

$N$, the number of civilizations in our Galaxy with which communication might be possible; $R_{*}$, the mean rate of star formation averaged over the lifetime of the Galaxy; $f_{p}$, the fraction of stars with planetary systems; $n_{e}$, the mean number of planets in each planetary system with environments favorable for the origin of life; $f_{l}$, the fraction of such favorable planets on which life does develop; $f_{i}$, the fraction of such inhabited planets on which intelligent life with manipulative abilities arises during the lifetime of their local sun; $f_{c}$, the fraction of planets populated by intelligent beings on which an advanced technical civilization arises during the host star's lifetime; $L$, the lifetime of the technical civilization.

There are reliable estimates for the first three factors $\left(R_{*} \cdot f_{p} \cdot n_{e} \sim 0.1^{7}\right)$ based on recent astronomical observations of exoplanets, protoplanetary disks, and star-forming regions. However, because we have not yet discovered any extraterrestrial life, nor elucidated the origins of terrestrial life, the other remaining factors are highly conjectural owing to the one-sample statistics of Earth.

\footnotetext{
${ }^{1}$ Department of Natural Science, Faculty of Science and Engineering, Tokyo City University, Setagaya, Tokyo 158-8557, Japan. ${ }^{2}$ Frontier Research Institute for Interdisciplinary Sciences, Tohoku University, Sendai, Miyagi 980-8578, Japan. email: ktsumura@tcu.ac.jp
} 


\begin{tabular}{|c|c|c|}
\hline Estimated value of $f_{i}$ & Estimated value of $f_{l} \cdot f_{i} \cdot f_{c}$ & Reference \\
\hline$\sim 0.1$ & $\sim 0.01$ & 6 \\
\hline$\sim 1$ & $0.1-0.2$ & 10 \\
\hline$\sim 1$ & $\sim 0.5$ & 11 \\
\hline$\sim 1$ & $\sim 1$ & 12 \\
\hline$\sim 1$ & $>0.1$ & 13 \\
\hline$\sim 1$ & 0.01 & 14 \\
\hline- & $<10^{-10}$ & 8 \\
\hline- & 0.01 & 15 \\
\hline 0.01 & $10^{-4}$ & 16 \\
\hline $0.01-0.1$ & - & \begin{tabular}{|l|}
17 \\
\end{tabular} \\
\hline 0.2 & 0.02 & 18 \\
\hline- & $>1.7 \cdot 10^{-11}$ & 19 \\
\hline 0.5 & 0.05 & 20 \\
\hline$\sim 1$ & $<10^{-40}$ & 21 \\
\hline$\sim 0.15$ & - & This work \\
\hline
\end{tabular}

Table 1. Previous estimates of $f_{i}$ and $f_{l} \cdot f_{i} \cdot f_{c}$.

We discuss $f_{i}$ among these conjectural factors in the Drake equation in this paper. Previous estimates of $f_{i}$ range from pessimistic $\left(f_{i} \sim 0\right)$ to optimistic $\left(f_{i} \sim 1\right)$. In general, while many physicists and astronomers prefer the optimistic value, many biologists prefer a value several orders of magnitude smaller ${ }^{8,9}$. Table 1 shows the various estimated values of $f_{i}$ and $f_{l} \cdot f_{i} \cdot f_{c}$ to date.

This paper introduces a new approach to estimating the probability that life on Earth has not gone extinct since the birth of life, $f_{i, \oplus}$. Since its birth, life on Earth has gone through many extinction events due to various random external factors, such as changes in the environment or impacts of meteorites. Extinction events of high intensity (where a significant fraction of species disappear) occur much less frequently than events of low intensity. The fossil record in the Phanerozoic Eon, which covers $540 \mathrm{Myr}$ to the present ${ }^{22,23}$, indicates that a histogram of extinction intensity can be well modeled by a log-normal distribution. This log-normal distribution

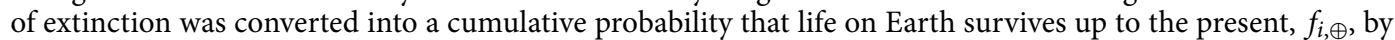
"continuing to win the lottery of extinction" since its birth. The obtained survival probability, $f_{i, \oplus}$, can be a template for estimating $f_{i}$ in the Drake equation, or other factors for estimating the number of life-bearing exoplanets, assuming that life on any other exoplanets essentially always becomes complex if it does not become extinct first.

\section{Histogram of the terrestrial extinction history}

Based on the Sepkoski's compendium ${ }^{22}$, a biodiversity database has been created from the Phanerozoic Eon fossil $\operatorname{record}^{23}$, and the study described in this paper is based on these data. Figure 1 (top) shows the number of known marine animal genera as a function of time for all data (black), and data with single occurrence and poorly dated genera removed (blue). The six major mass extinction events ${ }^{24,25}$, the Ordovician-Silurian extinction at 443.8 Myr ago (O-S), the Late Devonian extinction at 372.2 Myr ago (F-F), the Capitanian extinction at 259.8 Myr ago (Cap), the Permian-Triassic extinction at 251.9 Myr ago (P-T), the Tiassic-Jurassic extinction at 201.4 Myr ago (T-J), and the Cretaceous-Paleogene extinction at $66 \mathrm{Myr}$ ago (K-Pg), are clearly seen in Fig. 1 (top). Five of the six major mass extinctions were most likely related to flood-basalt volcanism, and one (K-Pg) to the massive impact of an asteroid ${ }^{24}$. Figure 1 (bottom) shows the extinction intensity as a function of time. Extinction intensity is defined as the fraction of well-resolved genera (those having both a distinct first and last appearance known to the stage level) present in the bin that are absent in the following bin. Two more big extinction events at around $500 \mathrm{Myr}$ ago, the End Botomian extinction (B) at $517 \mathrm{Myr}$ ago and the Dresbachian extinction (D) at $502 \mathrm{Myr}$ ago, are also visible in Fig. 1 (bottom). Although the details of these two extinctions are unclear due to the paucity of fossil records at that time, these data have also been analyzed without arbitrarily dismissing them in this work. Further details about these data are described by reference ${ }^{23}$.

A histogram of extinction intensity, constructed using the extinction intensity history data shown in Fig. 1 (bottom), is shown in Fig. 2. Although the provided data ${ }^{23}$ have a time bin size of $1 \mathrm{Myr}$, the time resolution of these data is closer to $\sim 3 \mathrm{Myr}$ because the peaks of the big extinctions are three bins wide. Although extinctions are most likely sudden events, the extinction peaks are spread out because the fossil record is incomplete (the Signor-Lipps effect ${ }^{26}$ ). Therefore, the frequency of the histogram (vertical axis in Fig. 2) was divided by three to match the time resolution of 3 Myr.

This histogram was then fitted with a log-normal distribution function $\varphi_{\ln }(x)$ :

$$
\varphi_{\ln }(x)=\frac{1}{\sqrt{2 \pi} \sigma x} \exp \left(-\frac{(\ln x-\mu)^{2}}{2 \sigma^{2}}\right)
$$

where $x$ denotes the extinction intensity as a random variable, and $\mu$ and $\sigma$ are free parameters in this distribution function. Since the histogram has a peak at $x \sim 0.05$, minor mass extinctions $(x<0.2)$, in addition to massive 

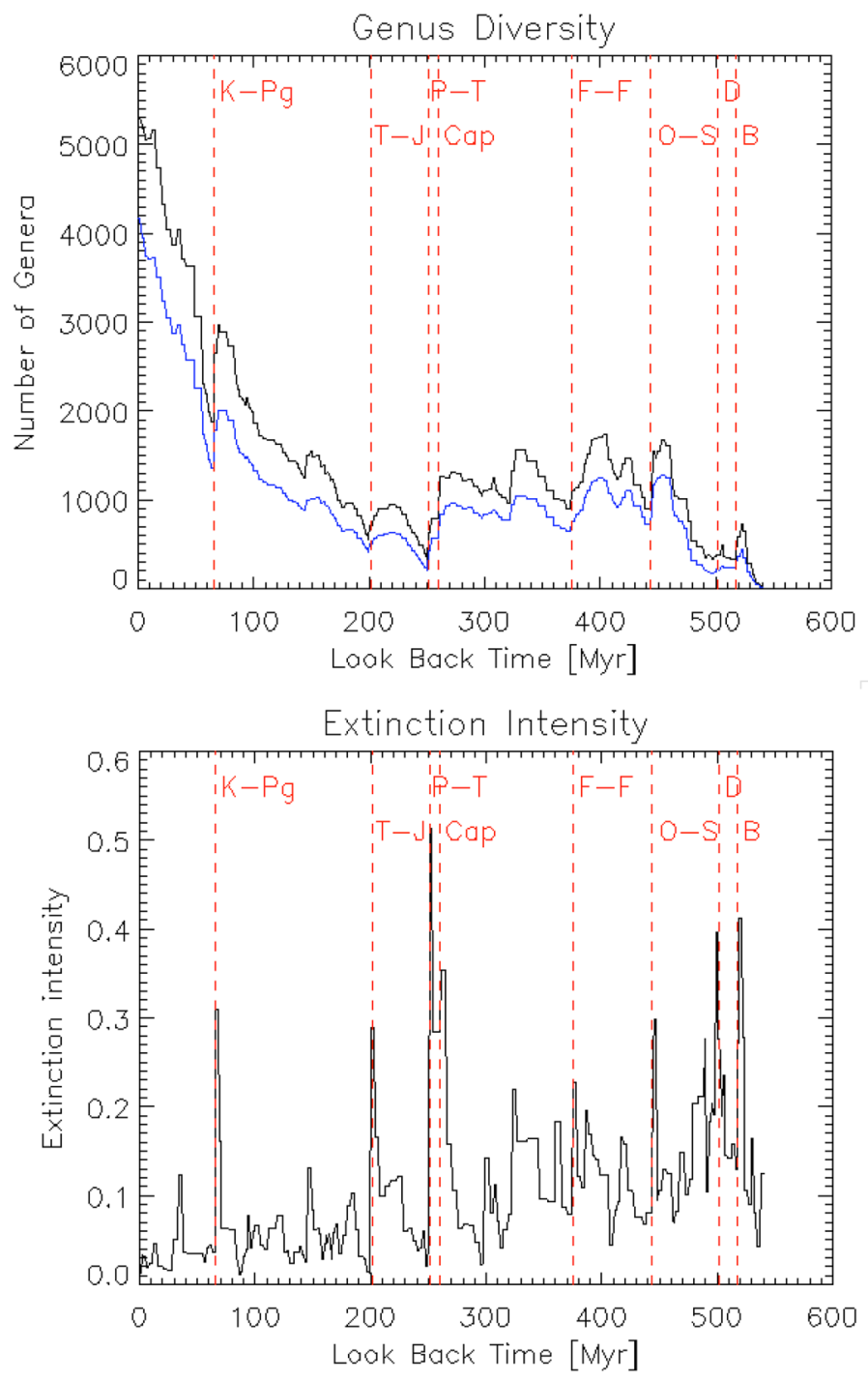

Figure 1. Biodiversity in the marine fossil record. Top: The number of known marine animal genera as a function of time for all data (black) and with single occurrence and poorly dated genera removed (blue). Bottom: Extinction intensity as a function of time. The six major mass extinctions (O-S, F-F, Cap, P-T, T-J, and $\mathrm{K}-\mathrm{Pg}$ ) and two more big extinctions (B and D) are visible. The data used in these figures are from reference ${ }^{23}$.

extinctions, affect the overall shape of this histogram. The choice of the log-normal distribution function is supported by the statistical principle that a random multiplicative process converges to a log-normal distribution owing to the central limit theorem. Since the terrestrial extinctions are caused by random events, such as volcanic activities $^{27-29}$, asteroid impacts ${ }^{30}$, superflares of the $\operatorname{Sun}^{31,32}$, gamma-ray bursts ${ }^{33}$, and so on, it is justified that the terrestrial extinction intensity can be expressed by a log-normal probability distribution as a result of these random multiplicative processes. The ultimate use of a log-normal distribution has precedence in the context of the Drake equation ${ }^{18,20,34}$.

The best-fitting parameters of the log-normal function fitted to the histogram are $\mu=-2.447$ and $\sigma=0.825$ with a scaling factor, and its reduced chi-squared $\left(\chi^{2}\right)$ is 0.988 . The best-fitting curve is shown in Figs. 2 and 3 (bottom) as a red line. Figure 3 (top) shows the confidence contour maps of the fitted parameters. The uncertainties associated with this fitting were evaluated as follows. First, all parameter sets within a $99 \%$ confidence level in the confidence contour map (Fig. 3 top) were extracted, and then envelops of the log-normal distribution functions with these extracted parameters are shown by dashed lines in Fig. 3 (bottom). Therefore, the two envelope curves in Fig. 3 (bottom) denote the uncertainties of this fitting with 99\% confidence level.

The same fitting procedures were applied to the histogram data using two additional distribution functions: the beta prime distribution function $\varphi_{\beta}(x)$ and the gamma distribution function $\varphi_{\gamma}(x)$ :

$$
\varphi_{\beta}(x)=\frac{1}{B(\alpha, \beta)} \frac{x^{\alpha-1}}{(1+x)^{\alpha+\beta}}
$$




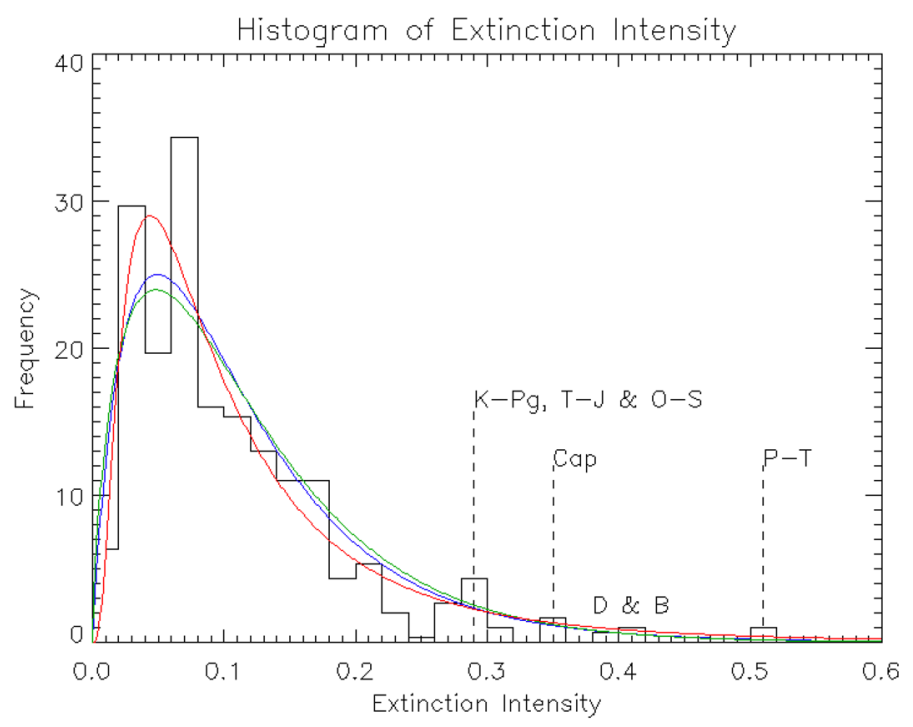

Figure 2. Histogram of extinction intensity. The lines show the best-fitting curves for a log-normal distribution function (red), beta prime distribution function (blue), and gamma distribution function (green).

$$
\varphi_{\gamma}(x)=\frac{\lambda^{k}}{\Gamma(k)} x^{k-1} e^{-\lambda x}
$$

where $B(\alpha, \beta)$ denotes the beta function with two free parameters of $\alpha$ and $\beta$, and $\Gamma(k)$ denotes the gamma function with two free parameters of $k$ and $\lambda$. These functions were previously used to estimate the factors in the Drake equation ${ }^{20}$. Figure 2 also shows the best-fitting curves of these distribution functions. The reduced $\chi^{2}$ of these fits for the beta prime distribution function and the gamma distribution function are 1.184 and 1.329, respectively. These poorer fitting statistics imply that the log-normal distribution function is more appropriate for the considered histogram, which lends credence to the assumption that the terrestrial extinction process is based on a random multiplicative process. Therefore, only the fitting results from the log-normal distribution function are considered hereafter.

\section{Estimation of the survival probability of terrestrial life}

The previous section showed that the histogram of extinction intensity in the Phanerozoic Eon could be well fitted by a log-normal distribution function (Figs. 2 and 3). In this section, a model is proposed to estimate the probability that life on Earth has not gone extinct from its birth to the present epoch. In this model, the histogram of extinction intensity is interpreted as a probability distribution, representing how many fractions of all genera become extinct within a unit timescale ( $3 \mathrm{Myr}$ ), if an extinction event of a certain magnitude, $x$, as a random variable with this probability distribution occurs every 3 Myr. Although $x$ was defined originally as the fraction of extinct genera, i.e., $0 \leq x \leq 1$, here we interpret $x$ as the magnitude of each extinction event, where $x=1$ means an extinction event with a minimal magnitude for all life on Earth to become extinct, and $x>1$ means extinction events with greater magnitudes than it. In this model, to illustrate the extinction history, this "extinction lottery" is drawn once every 3 Myr. If the result of this lottery is $x=0.05$, it means that $5 \%$ of the genera on Earth became extinct during this period, and then the next lottery is drawn in the next $3 \mathrm{Myr}$. If the extinction intensity $x$ takes a value of 1 or greater, it means all life on Earth are extinct, and the game is over. The fact that we are here now means that life on Earth has endured repeated extinction lotteries every 3 Myr since the birth of life (i.e. $x<1$ for all lotteries). Under such a condition, the probability that life on Earth has survived from the origin of life to the present, $f_{i, \oplus}$, is calculated below.

The cumulative distribution function, $\Phi_{\ln }(x)$, of the log-normal distribution function, $\varphi_{\ln }(x)$, can be expressed as:

$$
\Phi_{\ln }(x)=\int_{0}^{x} \varphi_{\ln }\left(x^{\prime}\right) d x^{\prime}=\frac{1}{2} \operatorname{erfc}\left(-\frac{\ln x-\mu}{\sqrt{2} \sigma}\right)
$$

where $\operatorname{erfc}(x)$ denotes the complementary error function. A value of $p=\Phi_{\ln }(1)$ means the probability that the extinction intensity, $x$, as a random variable takes a value smaller than 1 . This can be taken to mean that not all life on Earth becomes extinct; in other words, some genera on Earth have survived through a unit timescale ( 3 Myr). The history of evolution on Earth shows that life is quite resilient, because it eventually recovers even after big extinction events ${ }^{35}$. Therefore, life persist unless the result of the lottery is $x>1$. Since "winning an extinction lottery" is defined as the result of $x<1$, the probability of winning this extinction lottery is $p$. Thus, the survival probability $f_{i, \oplus}$ for duration $T$ can be expressed as a probability of winning $T / \Delta T$ times in the repeated extinction lotteries, 

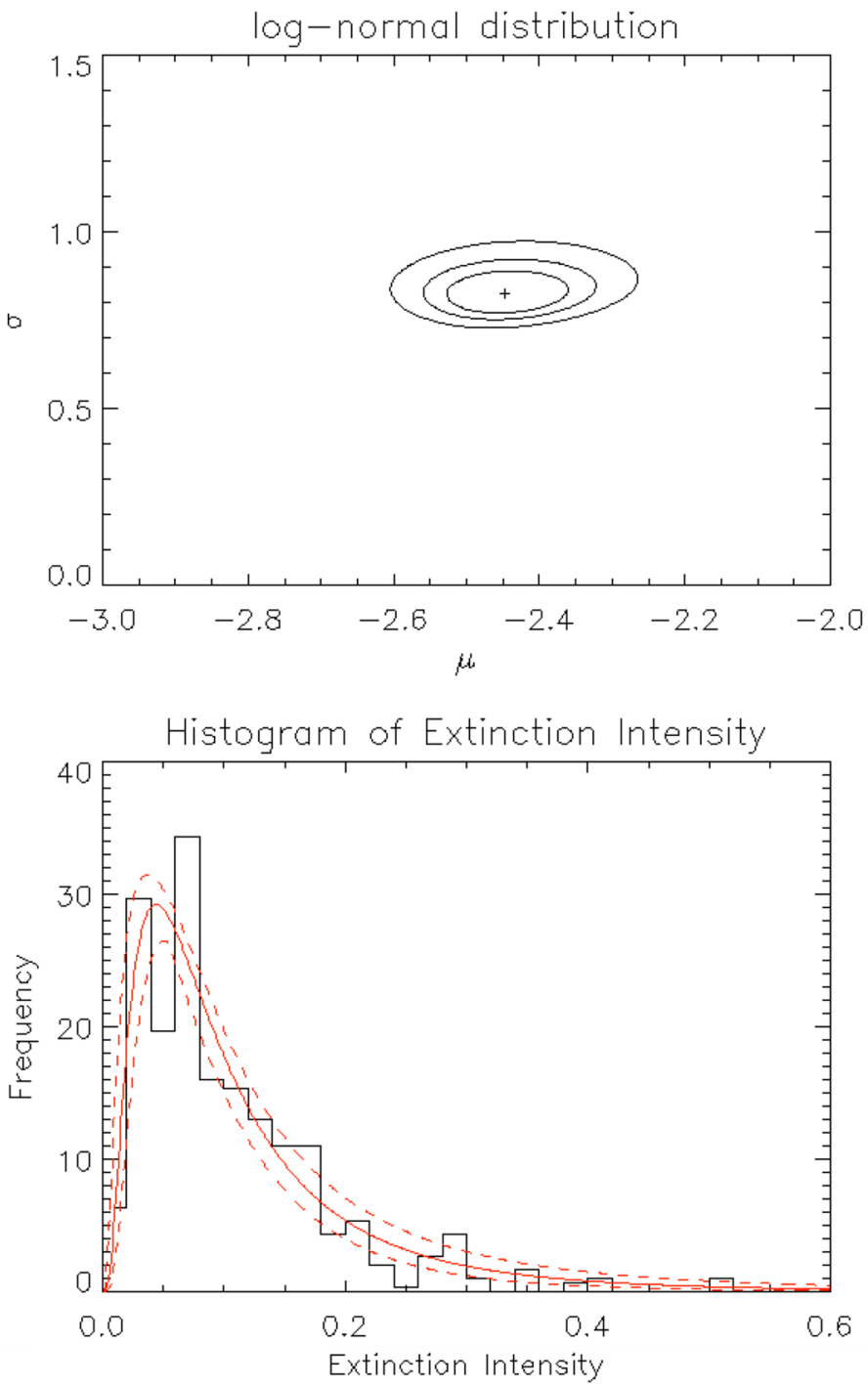

Figure 3. Fitting the histogram of extinction intensity with a log-normal distribution function. Top: Contour map of $\chi^{2}$ of the fitting. The cross mark at the center of the contour shows the best-fitting parameter set, and the three contour curves show $68 \%, 90 \%$, and $99 \%$ confidence levels, respectively. Bottom: Histogram of the extinction intensity and its best-fitting log-normal distribution function (red curve). The dashed red curves constrain the $99 \%$ confidence level region.

$$
f_{i, \oplus}(T)=p^{T / \Delta T}
$$

where $\Delta T$ is the time resolution of the probability distribution function $(\Delta T=3 \mathrm{Myr}$ in this case).

Using the best-fitting parameter set of $\mu$ and $\sigma$, the value of $p$ is calculated as $p=\Phi_{\ln }(1)=0.9985_{-0.0058}^{+0.0012}(99 \%$ confidence level), which means that the probability that some genera on Earth survive for $3 \mathrm{Myr}$ is $\sim 99.85 \%$, or the probability that all life on Earth becomes extinct during a 3 Myr period is $\sim 0.15 \%$. Therefore, the estimated survival probability of life on Earth during the Phanerozoic Eon $(T=540 \mathrm{Myr})$ is $f_{i, \oplus}(540 \mathrm{Myr})=0.76_{-0.06}^{+0.01}$. This means that life on Earth had a $\sim 24 \%$ probability of becoming extinct during the Phanerozoic Eon. This value is quite reliable because the log-normal distribution function was obtained by fitting to the histogram of the extinction history in the Phanerozoic Eon.

Recent geological evidence has suggested that life on Earth first occurred 3.7-4.1 Gyr ago ${ }^{36-41}$. Assuming that the value of $p$, obtained from the fossil record during the Phanerozoic Eon (540 Myr ago to present), can be extended to the entire history of life on Earth (3.7-4.1 Gyr ago to present), the survival probability for the entire history of life can be calculated as $f_{i, \oplus}(3.7 \mathrm{Gyr})=0.16_{-0.03}^{+0.01}$ or $f_{i, \oplus}(4.1 \mathrm{Gyr})=0.13_{-0.13}^{+0.01}$. Therefore, as a conclusion, the probability that life on Earth survived without becoming completely extinct can be estimated as $\sim 15 \%$ based on the fossil records of extinction from the Phanerozoic Eon. 


\section{Evaluation of the model assumptions}

Although the extinction intensity, $x$, is defined as the fraction of extinct genera, i.e. $0<x<1$, the histogram of $x$ was fitted by the log-normal distribution function $\varphi_{\ln }(x)$ defined in $0<x<\infty$. Therefore, one might think it is better to use a truncated log-normal distribution function defined in $0<x<1, \varphi_{\ln }^{\prime}(x)$ :

$$
\varphi_{\ln }^{\prime}(x)=\frac{1}{\Phi_{\ln }(1)-\Phi_{\ln }(0)} \varphi_{\ln }(x)
$$

The fitting procedure was conducted with the truncated log-normal distribution function and the same bestfitting parameters of $\mu=-2.447$ and $\sigma=0.825$ are obtained. Because the difference between $\varphi_{\ln }^{\prime}(x)$ and $\varphi_{\ln }(x)$ is only a scaling factor with a given parameter set of $\mu$ and $\sigma$, and the scaling factor is determined by fitting the function to the data, it is mathematically correct that the same best-fitting parameters are obtained with the $\log$-normal distribution function $\varphi_{\ln }(x)$ and the truncated $\log$-normal distribution function $\varphi_{\ln }^{\prime}(x)$. This result gains more credibility to employ the log-normal distribution function.

The obtained value of $f_{i, \oplus}$ represents the probability that life on Earth has survived various random extinction events since the birth of life to the present. One big assumption in this model is that the obtained extinction rate determined over only the last $540 \mathrm{Myr}$ (the Phanerozoic Eon) can be extended to the entire history of life on Earth ( $\sim 4 \mathrm{Gyr})$; however there is no guarantee that this assumption is correct. For example, Earth experienced the Late Heavy Bombardment (LHB) 3.8-3.9 Gyr ago, which likely destroyed almost all life present on Earth at that time ${ }^{42-45}$, but this truly massive extinction event was not included in the model. In addition, even within the Phanerozoic Eon, the extinction rate declines with time ${ }^{46}$, which can be seen in Fig. 1 (bottom). Moreover, the dataset used in this model (Fig. 1) was constructed using fossil records of marine animal genera, not all types of life $^{23}$. Therefore, it is not clear whether the modern extinction rate of marine animal genera, which is modeled in this paper, can be applied to all types of life across all of history of Earth. In this paper, however, we assumed that the modern extinction rate of marine animal genera could be applied to all life throughout history. This is a big assumption, but this is the best that can be made at this point with the available data.

The purpose of the Drake equation is to deal specifically with questions of if, how, when and how often evolution leads to complex life, which cannot be answered completely without other examples of evolution. Therefore, by using the terrestrial history as a template for the histories of life on exoplanets, we have attempted to provide some useful perspective using the available information. In this context, it was assumed that the obtained survival probability, $f_{i, \oplus}$, can be used to represent $f_{i}$ in the Drake equation, i.e., $f_{i}=f_{i, \oplus} \sim 0.15$, because the only available data pertain to the history of Earth. This assumption means that the evolutionary history of Earth is universal, i.e., once the origin of life is accomplished, the evolution of complex life always take place in any stable, sufficiently extensive environment if it does not become extinct first ${ }^{47}$. This assumption is called the "Planet of the Apes" hypothesis ${ }^{9}$ or the astrobiological Copernican principle ${ }^{48}$. A unique point of the method used here was to model extinction, rather than the appearance of life, to address the Drake equation.

\section{Application to other estimations}

According to Equation (6), the survival probability of life on Earth, $f_{i, \oplus}$, has two parameters: $p$ (the survival probability for a time-bin of $\Delta T=3 \mathrm{Myr}$ ) and $T$ (the evolution duration from the birth of life to the emergence of intelligent life). Assuming that values for Earth, $p=0.9985$ and $T \sim 4 \mathrm{Gyr}$, are universal, $f_{i}=f_{i, \oplus} \sim 0.15$ was obtained. This expression of $f_{i}$ has a room for adjusting these two parameters for other life-bearing exoplanets to match their local environments and situations. For example, some exoplanets have harsher environments than Earth, which would require any life to ensure stronger stellar winds or interstellar radiation fields than those in our Solar system; hence we can readjust the survival probability so that $p$ has a smaller value. If the evolution speed of life on some exoplanets is slower than on Earth, we can apparently readjust the evolution time duration so that $T$ is larger. It is still difficult to estimate $p$ and $T$ for other exoplanets, but it can provide some useful constraints of $f_{i}$ based on scientific observations of exoplanets.

This approach can also be extended to the Seager equation, a parallel version of the Drake equation ${ }^{49}$. The Seager equation estimates the number of planets with detectable signs of life by way of biosignature gases as:

$$
N^{\prime}=N_{*} \cdot f_{Q} \cdot f_{H Z} \cdot f_{O} \cdot f_{L} \cdot f_{S}
$$

$N^{\prime}$, the number of planets with detectable signs of life by way of biosignature gases; $N_{*}$, the number of stars in the survey; $f_{Q}$, the fraction of stars in the survey that are suitable for planet finding (e.g., quiet non-variable stars or non-binary stars); $f_{H Z}$, the fraction of stars with rocky planets in the habitable zone; $f_{O}$, the fraction of those planets that can be observed, according to limitations of planet orbital geometry or other limiting factors; $f_{L}$, the fraction of planets that have life; $f_{S}$, the fraction of planets with life that produce a detectable biosignature gas by way of a spectroscopic signature.

The model introduced in this study can be applied to estimate $f_{S}$ in the Seager equation. Here, we consider molecular oxygen $\left(\mathrm{O}_{2}\right)$ as the favored biosignature gas, which is a gas produced by life that can accumulate to detectable levels in an exoplanetary atmosphere. The permanent rise to measurable concentrations of $\mathrm{O}_{2}$ in the atmosphere of Earth via photosynthesis of prokaryotic and eukaryotic organisms in the ocean, known as the Great Oxidation Event (GOE), occurred around 2.4 Gyr ago ${ }^{50,51}$. Therefore, Earth took 1.3-1.7 Gyr from the birth of life to be detectable by astronomical spectroscopic observations of biosignature gas by outside observers. The probability that life on Earth survives until the GOE, $f_{S, \oplus}$, can be calculated using the same method shown in Equation (6), which yields $f_{S, \oplus}(1.3 \mathrm{Gyr})=0.52_{-0.06}^{+0.01}$ and $f_{S, \oplus}(1.7 \mathrm{Gyr})=0.42_{-0.06}^{+0.01}$. Assuming again that the time taken for photosynthesis to evolve is the same as that on Earth, these values can be interpreted as $f_{S}$ in the Seager equation. A value of $f_{S}=0.5$ was originally speculated ${ }^{49}$, which is a reasonable estimate. 
This model also can be applied to estimate the probability that existing life, including human beings on Earth, would become extinct before Earth became inhabitable. As the Sun brightens due to its natural evolutionary process, Earth will become uninhabitable in the far future due to rising temperatures. According to one model, the complete loss of oceans of Earth may occur in little over 2 Gyr from present, thereby transforming Earth into a desert planet. This suggests that most forms of life will unable to survive for more than $1.3 \mathrm{Gyr}$ from the present day on Earth $^{52}$. The survival probability of a 1.3-Gyr period in our model is $f_{i, \oplus}(1.3 \mathrm{Gyr}) \sim 0.5$, indicating that existing life on Earth, including humans, have a $50 \%$ probability of becoming extinct before Earth becomes inhabitable. This survival probability is much higher than the estimated total longevity of our species of 0.2 million to 8 million years at the $95 \%$ confidence level under the Copernican principle ${ }^{53}$. This difference may occur because advanced civilizations would be affected by much smaller catastrophes that would not show up as mass extinctions in the geological records. For example, asteroid impactors with 1-km diameters (making a $20-\mathrm{km}$ diameter crater) are expected to occur about every $10^{5}$ years $^{54}$ and large volcanic eruptions that could cause "volcanic winter" are expected to occur about every $5 \times 10^{4}$ year $^{55}$; both types of events are capable of destroying or greatly affecting an advanced civilization. Such s discussion, however, is more related to the factors $f_{c}$ and $L$ in the Drake equation, rather than $f_{i}$ estimated in this paper, which are critical parameters to be considered when searching for intelligent civilizations.

\section{Conclusions}

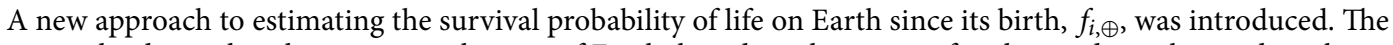
principle idea is that the extinction history of Earth, based on the marine fossil record, can be used to obtain the survival probability of life since it began on Earth. The obtained value is $f_{i, \oplus} \sim 0.15$. Under the astrobiological Copernican principle ${ }^{48}$, this survival probability can be interpreted as $f_{i}$ in the Drake equation, i.e., $f_{i}=f_{i, \oplus} \sim 0.15$. Because $f_{i, \oplus}$ is a two-parameter function of $p$ (the survival probability for a unit time) and $T$ (the evolution time from the birth of life to intelligent life), this method can be extended to estimate the survival probability on other life-bearing exoplanets by adjusting these two parameters to the local environments of the considered exoplanets.

Received: 26 April 2020; Accepted: 20 July 2020

Published online: 30 July 2020

\section{References}

1. Wolszczan, A. \& Frail, D. A. A planetary system around the millisecond pulsar PSR1257+12. Nature 355, 145-147. https://doi. org/10.1038/355145a0 (1992).

2. Mayor, M. \& Queloz, D. A Jupiter-mass companion to a solar-type star. Nature 378, 355-359. https://doi.org/10.1038/378355a0 (1995).

3. Siemion, A. et al. Searching for extraterrestrial intelligence with the square kilometre array. In Advancing Astrophysics with the Square Kilometre Array (AASKA14), 116. https://doi.org/10.22323/1.215.0116 (2015).

4. Zhang, Z.-S. et al. First SETI observations with China's five-hundred-meter aperture spherical radio telescope (FAST). Astrophys. J. 891, 174. https://doi.org/10.3847/1538-4357/ab7376 (2020).

5. Drake, F. D. Project Ozma. Phys. Today 14, 40-46. https://doi.org/10.1063/1.3057500 (1961).

6. Sagan, C. Direct contact among galactic civilizations by relativistic interstellar spaceflight. Planet. Space Sci. 11, 485-498. https:// doi.org/10.1016/0032-0633(63)90072-2 (1963).

7. Prantzos, N. A probabilistic analysis of the Fermi paradox in terms of the Drake formula: the role of the L factor. Mon. Not. R. Astron. Soc. 493, 3464-3472. https://doi.org/10.1093/mnras/staa512 (2020).

8. Tipler, F. J. Extraterrestrial intelligent beings do not exist. Q. J. R. Astron. Soc. 21, 267-281 (1980).

9. Lineweaver, C. H. Paleontological tests: Human-like intelligence is not a convergent feature of evolution. In From Fossils to Astrobiology: Records of Life on Earth and Search for Extraterrestrial Biosignatures (eds Seckbach, J. \& Walsh, M.) 353-368 (Springer, Dordrecht, 2008). https://doi.org/10.1007/978-1-4020-8837-7_17.

10. Pearman, J. P. T. Extraterrestrial intelligent life and interstellar communication: An informal discussion. In Interstellar communication. The search for extraterrestrial life (ed. Cameron, A. G. W.) 287-293 (W.A. Benjamin Inc, New York, 1963).

11. Cameron, A. G. W. Communicating with intelligent life on other worlds. Sky Telesc. 26, 258 (1963).

12. Drake, F. D. The radio search for intelligent extraterrestrial life. In Current Aspects of Exobiology (eds Mamikunian, G. \& Briggs, M.) 323-345 (Springer, Berlin, 1965). https://doi.org/10.1016/B978-1-4832-0047-7.50015-0.

13. Oliver, B. M. Proximity of galactic civilizations. Icarus 25, 360-367. https://doi.org/10.1016/0019-1035(75)90031-7 (1975).

14. Freeman, J. \& Lampton, M. Interstellar archaeology and the prevalence of intelligence. Icarus 25, 368-369. https://doi. org/10.1016/0019-1035(75)90032-9 (1975).

15. Wallenhorst, S. G. The Drake equation reexamined. Q. J. R. Astron. Soc. 22, 380 (1981).

16. Drake, F. \& Sobel, D. Is anyone out there? The scientific search for extraterrestrial intelligence (Delacorte Press, New York, 1992).

17. Forgan, D. H. A numerical testbed for hypotheses of extraterrestrial life and intelligence. Int. J. Astrobiol. 8, 121-131. https://doi. org/10.1017/S1473550408004321 (2009).

18. Maccone, C. The statistical Drake equation. Acta Astronautica 67, 1366-1383. https://doi.org/10.1016/j.actaastro.2010.05.003 (2010).

19. Frank, A. \& Sullivan, I. W. T. A new empirical constraint on the prevalence of technological species in the universe. Astrobiology 16, 359-362. https://doi.org/10.1089/ast.2015.1418 (2016).

20. Bloetscher, F. Using predictive Bayesian Monte Carlo-Markov Chain methods to provide a probablistic solution for the Drake equation. Acta Astronautica 155, 118-130. https://doi.org/10.1016/j.actaastro.2018.11.033 (2019).

21. Totani, T. Emergence of life in an inflationary universe. Sci. Rep. 10, 1671. https://doi.org/10.1038/s41598-020-58060-0 (2020).

22. Sepkoski, J. J. A compendium of fossil marine animal genera. Bull. Am. Paleontol. 363, 1-560 (2002).

23. Rohde, R. A. \& Muller, R. A. Cycles in fossil diversity. Nature 434, 208-210. https://doi.org/10.1038/nature03339 (2005).

24. Rampino, M., Caldeira, K. \& Prokoph, A. What causes mass extinctions? Large asteroid/comet impacts, flood-basalt volcanism, and ocean anoxia-Correlations and cycles. Geol. Soc. Am. Spec. Paper 542, 271-302. https://doi.org/10.1130/2019.2542(14) (2019).

25. Rampino, M. R. \& Shen, S.-Z. The end-Guadalupian (259.8 Ma) biodiversity crisis: the sixth major mass extinction?. Hist. Biol.. https://doi.org/10.1080/08912963.2019.1658096 (2019).

26. Signor, P. \& Lipps, J. Sampling bias, gradual extinction patterns and catastrophes in the fossil record. Geol. Soc. Am. Spec. Paper 190, 291-296. https://doi.org/10.1130/SPE190 (1982). 
27. Hesselbo, S. P., Robinson, S. A., Surlyk, F. \& Piasecki, S. Terrestrial and marine extinction at the Triassic-Jurassic boundary synchronized with major carbon-cycle perturbation: A link to initiation of massive volcanism?. Geology 30, 251-254. https://doi. org/10.1130/0091-7613(2002)030<0251:TAMEAT>2.0.CO;2 (2002).

28. Kamo, S. L. et al. Rapid eruption of Siberian flood-volcanic rocks and evidence for coincidence with the Permian-Triassic boundary and mass extinction at 251 Ma. Earth Planet. Sci. Lett. 214, 75-91. https://doi.org/10.1016/S0012-821X(03)00347-9 (2003).

29. Jones, D. S., Martini, A. M., Fike, D. A. \& Kaiho, K. A volcanic trigger for the Late Ordovician mass extinction? Mercury data from south China and Laurentia. Geology 45, 631-634. https://doi.org/10.1130/G38940.1 (2017).

30. Schulte, P. et al. The Chicxulub asteroid impact and mass extinction at the cretaceous-paleogene boundary. Science 327, $1214-1218$. https://doi.org/10.1126/science.1177265 (2010).

31. LaViolette, P. Evidence for a solar flare cause of the pleistocene mass extinction. Radiocarbon 53, 303-323. https://doi.org/10.1017/ S0033822200056575 (2011).

32. Lingam, M. \& Loeb, A. Risks for life on habitable planets from superflares of their host stars. Astrophys. J. 848, 41. https://doi. org/10.3847/1538-4357/aa8e96 (2017).

33. Melott, A. L. et al. Did a gamma-ray burst initiate the late Ordovician mass extinction?. Int. J. Astrobiol. 3, 55-61. https://doi. org/10.1017/S1473550404001910 (2004).

34. Maccone, C. SETI and SEH (statistical equation for habitables). Acta Astronautica 68, 63-75. https://doi.org/10.1016/j.actaa stro.2010.06.010 (2011).

35. Chen, Z.-Q. \& Benton, M. J. The timing and pattern of biotic recovery following the end-Permian mass extinction. Nat. Geosci. 5, 375-383. https://doi.org/10.1038/ngeo1475 (2012).

36. Mojzsis, S. J. et al. Evidence for life on Earth before 3,800 million years ago. Nature 384, 55-59. https://doi.org/10.1038/384055a0 (1996).

37. Rosing, M. T. ${ }^{13} \mathrm{C}$-depleted carbon microparticles in > 3700-Ma sea-floor sedimentary rocks from West Greenland. Science 283, 674. https://doi.org/10.1126/science.283.5402.674 (1999).

38. van Zuilen, M. A., Lepland, A. \& Arrhenius, G. Reassessing the evidence for the earliest traces of life. Nature 418, 627-630. https ://doi.org/10.1038/nature00934 (2002).

39. Ohtomo, Y., Kakegawa, T., Ishida, A., Nagase, T. \& Rosing, M. T. Evidence for biogenic graphite in early Archaean Isua metasedimentary rocks. Nat. Geosci. 7, 25-28. https://doi.org/10.1038/ngeo2025 (2014).

40. Bell, E. A., Boehnke, P., Harrison, T. M. \& Mao, W. L. Potentially biogenic carbon preserved in a 4.1 billion-year-old zircon. Proc. Natl. Acad. Sci. 112, 14518-14521. https://doi.org/10.1073/pnas.1517557112 (2015).

41. Pearce, B. K. D., Tupper, A. S., Pudritz, R. E. \& Higgs, P. G. Constraining the Time Interval for the Origin of Life on Earth. Astrobiology 18, 343-364. https://doi.org/10.1089/ast.2017.1674 (2018).

42. Cohen, B. A., Swindle, T. D. \& Kring, D. A. Support for the lunar cataclysm hypothesis from lunar meteorite impact melt ages. Science 290, 1754-1756. https://doi.org/10.1126/science.290.5497.1754 (2000).

43. Nisbet, E. G. \& Sleep, N. H. The habitat and nature of early life. Nature 409, 1083-1091. https://doi.org/10.1038/35059210 (2001).

44. Line, M. A. The enigma of the origin of life and its timing. Microbiology 148, 21-27. https://doi.org/10.1099/00221287-148-1-21 (2002).

45. Zahnle, K. et al. Emergence of a habitable planet. In Geology and Habitability of Terrestrial Planets (eds Fishbaugh, K. E. et al.) 35-78 (Springer, New York, 2007). https://doi.org/10.1007/978-0-387-74288-5_3.

46. MacLeod, N. The Great Extinctions: What Causes Them and how They Shape Life (Firefly Books, Richmond Hill, 2013).

47. Bains, W. \& Schulze-Makuch, D. The cosmic zoo: The (near) inevitability of the evolution of complex, macroscopic life. Life 6, 25. https://doi.org/10.3390/life6030025 (2016).

48. Westby, T. \& Conselice, C. J. The astrobiological copernican weak and strong limits for intelligent life. Astrophys. J. 896, 58. https ://doi.org/10.3847/1538-4357/ab8225 (2020).

49. Seager, S. The search for habitable planets with biosignature gases framed by a 'Biosignature Drake Equation'. Int. J. Astrobiol. 17, 294-302. https://doi.org/10.1017/S1473550417000052 (2018).

50. Sessions, A. L., Doughty, D. M., Welander, P. V., Summons, R. E. \& Newman, D. K. The continuing puzzle of the great oxidation event. Curr. Biol. 19, R567-R574. https://doi.org/10.1016/j.cub.2009.05.054 (2009).

51. Lyons, T. W., Reinhard, C. T. \& Planavsky, N. J. The rise of oxygen in Earth's early ocean and atmosphere. Nature 506, $307-315$. https://doi.org/10.1038/nature13068 (2014).

52. Wolf, E. T. \& Toon, O. B. The evolution of habitable climates under the brightening Sun. J. Geophys. Res. Atmos. 120, 5775-5794. https://doi.org/10.1002/2015JD023302 (2015).

53. Gott, J. R. Implications of the Copernican principle for our future prospects. Nature 363, 315-319. https://doi.org/10.1038/36331 $5 \mathrm{a} 0$ (1993).

54. Chapman, C. R. \& Morrison, D. Impacts on the Earth by asteroids and comets: assessing the hazard. Nature 367, 33-40. https:// doi.org/10.1038/367033a0 (1994).

55. Rampino, M. R. Supereruptions as a threat to civilizations on earth-like planets. Icarus 156, 562-569. https://doi.org/10.1006/ icar.2001.6808 (2002).

\section{Acknowledgements}

The author would like to thank Yasuhisa Nakajima (Tokyo City University) for discussions regarding paleontology, and Enago (www.enago.jp) for the English language review. This research was supported by JSPS KAKENHI Grant Numbers 18KK0089 and 20H04744.

\section{Author contributions}

K.T. performed all work in this paper.

\section{Competing interests}

The author declares no competing interests.

\section{Additional information}

Correspondence and requests for materials should be addressed to K.T.

Reprints and permissions information is available at www.nature.com/reprints.

Publisher's note Springer Nature remains neutral with regard to jurisdictional claims in published maps and institutional affiliations. 
(c) (i) Open Access This article is licensed under a Creative Commons Attribution 4.0 International cc) License, which permits use, sharing, adaptation, distribution and reproduction in any medium or format, as long as you give appropriate credit to the original author(s) and the source, provide a link to the Creative Commons license, and indicate if changes were made. The images or other third party material in this article are included in the article's Creative Commons license, unless indicated otherwise in a credit line to the material. If material is not included in the article's Creative Commons license and your intended use is not permitted by statutory regulation or exceeds the permitted use, you will need to obtain permission directly from the copyright holder. To view a copy of this license, visit http://creativecommons.org/licenses/by/4.0/.

(C) The Author(s) 2020 